\title{
MicroRNA Target Signatures in Advanced Stage Neuroblastoma
}

\author{
Victoria R. Chu and Inhan Lee \\ miRcore \\ U.S.A.
}

\section{Introduction}

Neuroblastoma is a solid cancer that arises from precursor cells of the sympathetic nervous system. It is known for its extreme heterogeneity, ranging from spontaneous regression to rapid progression to metastasis. Prognostic factors include age and genetic abnormalities, with greater likelihood of survival in infants, suggesting that neuroblastoma is a developmental disorder (Jiang et al., 2011). Considering that neuroblastoma accounts for $15 \%$ of all cancer deaths among children, novel therapeutic targets are clearly needed. Furthermore, early differentiation of children who will not develop advanced-stage neuroblastoma would reduce long-term side effects due to treatment, improving quality of life of survivors. Since neuroblastoma arises from precursor cells, understanding the mechanism by which it becomes advanced-stage cancer might also provide insight into how normal stem cells transform into cancer stem cells. This chapter examines factors differentiating advanced, metastatic neuroblastoma from low-risk, localized neuroblastoma.

Common genetic abnormalities in neuroblastoma include the genetic amplification (multiple DNA copies) of MYCN, chromosome 1p36 and 11q23 deletion, and the gain of chromosome 17q22 (review in Jiang et al., 2011). Since all of these genetic abnormalities correlate with poor clinical outcome, they have been investigated as potential driving factors in advanced stage neuroblastoma. However, as advanced neuroblastoma also occurs without such genetic abnormalities, the signatures of advanced neuroblastoma must be determined to understand the mechanism of its progression. For example, though amplification of transcription factor MYCN was linked early on with a poor outcome (Bordow et al., 1998), MYCN status cannot fully account for all advanced-stage cases. In addition to DNA-level abnormalities, expression level changes of transcripts in advanced stage have been studied to accurately predict the disease outcome (Asgharzadeh et al., 2006; Oberthuer et al., 2006). However, MYCN transcript levels could not dependably identify advanced stage neuroblastoma either. It is clear that multiple gene regulation should be considered in deciphering neuroblastoma progression (De Preter et al., 2010; Overthuer et al., 2010). To differentiate the underlying mechanism of progression, several studies have assessed DNA abnormality and transcription level changes together, comparing, for example, all 
transcription levels of advanced-stage neuroblastoma with and without MYCN amplification. Recent research on transcript-level changes has incorporated microRNA expression changes (Lin et al., 2010).

MicroRNAs (miRNAs) are endogenous regulatory RNAs ranging in size from 16 to 25 nucleotides that are processed from hairpin-structured precursors by the enzyme Dicer (Kim, 2005). Once a mature single strand miRNA becomes part of a miRNARiboNucleoProtein complex (miRNP), it mostly functions as a negative regulator of other genes' protein production by directly interacting with the target genes' mRNAs in sequencecomplementary fashion. Though originally identified in terms of its translational repression function in animal, it also helps degrade target mRNAs themselves. So far, more than a thousand miRNAs have been identified in human (http://mirbase.org) (Kozomara \& Griffiths-Jones, 2010), some of them potentially regulating about 200 genes (Lim et al., 2005). As with transcription factors and other endogenous genes with regulatory roles, expression of each miRNA is strictly controlled and exhibits characteristic patterns based on biological context, such as tissue type or developmental stages. In line with the critical roles of miRNAs in biological processes, their deregulation is frequently observed in many diseases, including neuroblastoma.

Neuroblastoma-related miRNAs have been researched mainly in regard to two biological processes, anti-apoptosis (tumorigenesis), and epithelial mesenchymal transition (EMT: metastasis related). TP53 is a well-known transcription factor that induces apoptosis and miR-34 family transcription (He et al., 2007). In neuroblastoma, TP53 mutation is uncommon, whereas deletion on chromosome 1p36 (where mir-34a is located) frequently occurs. Another frequent deletion site of $11 \mathrm{q} 23$ coincides with mir-34b and mir-34c chromomal positions, implying the importance of the mir-34 family in neuroblastoma. On the other hand, MYCN amplification is frequent in neuroblastoma and several miRNAs are induced by MYCN, including the mir-17-92 cluster, which has been shown to be significantly upregulated in MYCN-amplified cells (Schulte et al., 2008) and has been identified with a direct anti-proliferation function (Matsubara et al., 2007). In neuroblastoma, upregulation of the mir-17-92 cluster is correlated with the obstruction of the TGF-beta signaling pathway, thereby preventing apoptosis (Mestdagh et al., 2010a). MYCN also achieves anti-apoptotic function by downregulating other miRNAs which prevent cell proliferation. Low levels of MYCN allow for the over-expression of miR-184, which has been shown to reduce neuroblastoma tumor growth (Foley et al., 2010; Tivnan et al., 2010). While MYCN affects transcripton of certain miRNAs, some miRNAs can downregulate MYCN translation, such as miR-34 (Christoffersen et al., 2010). let-7 and miR-101 are also found to target MYCN and inhibit proliferation of MYCN-amplified neuroblastoma cells (Buechner et al., 2011). Similarly, Foley et al. demonstrate that miR-10a and miR-10b indirectly downregulate MYCN by targeting NCOR2, though the intermediate processes between NCOR2 and MYCN need further study (Foley et al., 2011). Therefore, miR-34 family deletion and MYCN amplification in neuroblastoma together respond to tumorigenesis through anti-apoptotic process.

Metastasis may also be brought on by altered levels of miRNA. Guo et al. show that 54 different miRNAs are significantly altered in metastatic as opposed to primary neuroblastoma tumors (Guo et al., 2010). A more specific study showed that overexpression of miR-524-5p decreases the invasive potential of neuroblastoma cells (Bray et al., 2011). 
Recently, the miR-34 family has been identified as blocking EMT by downregulating SNAI1 (Kim et al., 2011), showing its dual function of blocking tumorigenesis and metastasis. Haug et al. found that miR-92 downregulates tumor suppressor DKK3 (Haug et al, 2011), which normally inhibits the Wnt signaling pathway. The canonical Wnt signaling can induce EMT mostly by degrading E-cadherin (Heuberger \& Birchmeier, 2010). All this implies that a large network stretching across miR-34, MYCN, and the miR-17-92 cluster is responsible for both tumorogenesis and metastasis in neuroblastoma (Fig. 1). Hence, miRNAs hold promise as biomarkers for diagnostic and therapeutic purposes.

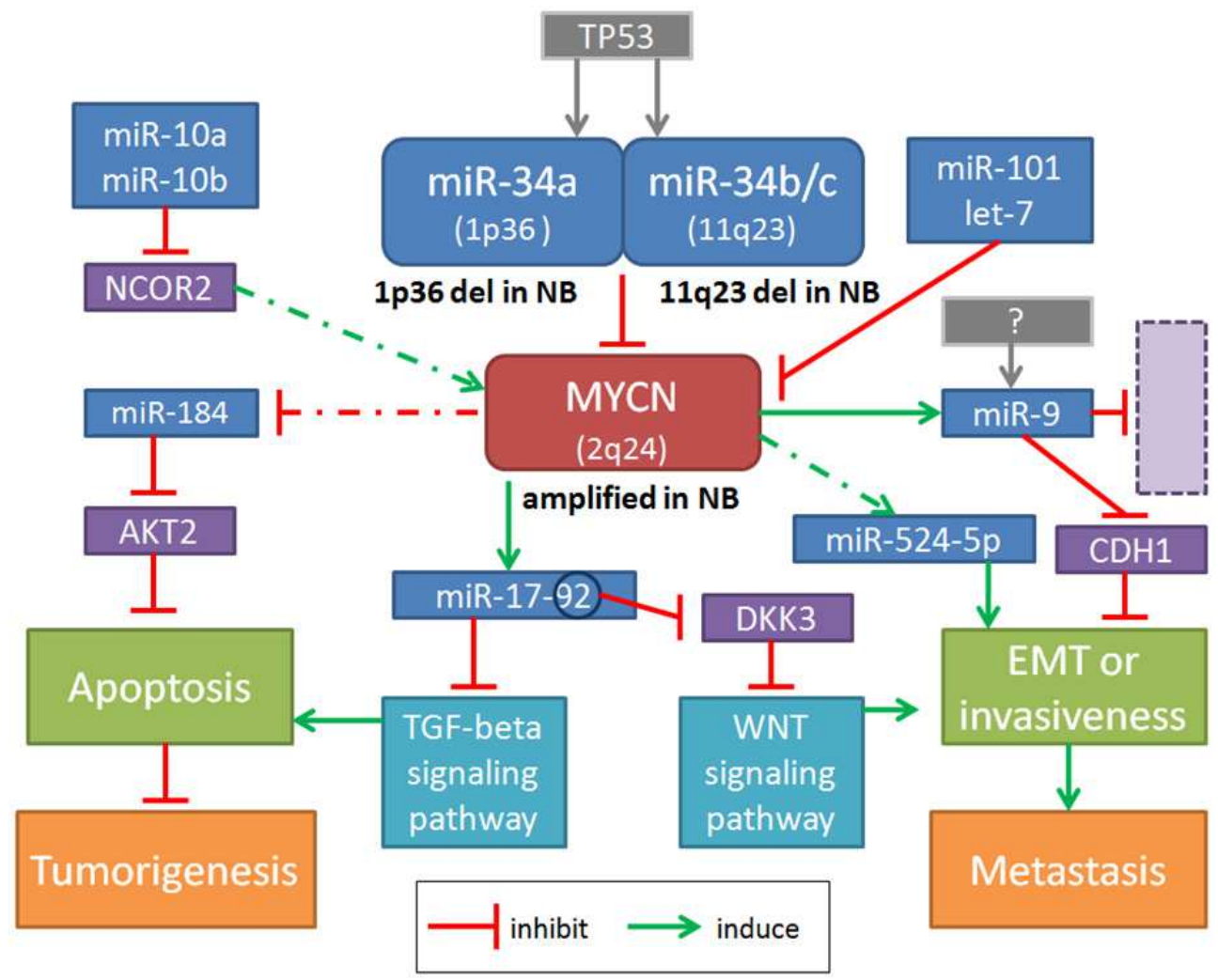

Fig. 1. Schematic diagram of miRNA network in neuroblastoma tumorigenesis and metastasis, including miR-9 from this study. The dotted box represents our six predicted targets in this study.

The problem remains that miRNAs' small size and similar sequences often make their global expression data noisier than mRNA data. Given that miRNAs regulate the expression of target mRNAs, correlating miRNA with mRNA expression could increase biomarker 
signals, leading to biomarkers with superior resolution and potentially revealing master regulators. To realize this potential, we need accurate miRNA target identification. While other areas of miRNA research have undergone continuous development, target prediction has lagged behind, partly due to the imperfect miRNA-mRNA bindings found in animals. As experimental and evolutionary evidence indicates that the $5^{\prime}$-end of miRNAs (the nucleotide position 1-8 at the $5^{\prime}$-end, also called the seed sequence) is important for recognition of target sequences in $3^{\prime}$-UTRs (untranslated regions) of mRNA, many computational algorithms utilize only 6-8 nucleotides (nt) of the $\sim 22$ mer miRNA to predict target mRNAs. However, there exist many genome-wide sequences matching this short stretch of 6-8 nt, resulting in a large number of false positives among the predicted targets and thus invalidating miRNA-mRNA correlations. Although a database of miRNAs and their experimentally verified targets exists (Papadopoulos et al., 2009), the list therein is still far from comprehensive.

We have identified a new class of miRNA targets, called miBridge, in which the $5^{\prime}$ - and $3^{\prime}$ end of a miRNA can simultaneously interact with the $3^{\prime}$-UTR and $5^{\prime}$-UTR of a single mRNA (Lee et al., 2009). We will use the terms "3UTR:5emiR" (a miRNA seed sequence and its target site on the 3'-UTR: conventional knowledge) and "5UTR:3emiR" (the 3 '-end of a miRNA - the other side of the seed region-and its recognition site in the 5'-UTR: our finding). Whereas other studies have not found global miRNA interactions with the $5^{\prime}$-UTR due to searching against the seed region (5UTR:5emiR), we have found significant 5UTR:3emiR interactions (Ajay, et al., 2010; Lee et al., 2009). This sequence-specific miRNA function through 5UTR:3emiR has been experimentally validated (Lee et al., 2009). In addition to these previous reports establishing the validity and utility of miBridge, we have recently generated a list of genome-wide miBridge targets using version 1 (v.1) prediction parameters. Interestingly, most known miRNA and mRNA (both 5'-UTR and 3'-UTR annotated) have miBridge interactions. Considering both ends of miRNA in the target prediction, miBridge targets are usually fewer in number (about $1 / 2$ to $1 / 10$ of other predictions), while having specific functions which align well with previous expresimental data.

In this chapter, we introduce a new approach to identifying miRNAs which differentiates high-risk (advanced) from low-risk neuroblastoma based on expression data and miBridge target predictions. When we applied the method to neuroblastoma patient datasets of mRNA and miRNA expression levels obtained from the Vandesompele group (Mestdagh et al., 2010b), we found miR-9 to be a consistent biomarker, all six predicted targets being downregulated and their expressions negatively correlated with miR-9 expression. This is interesting since MYCN is one of miR-9's transcription factors, though our analysis did not consider MYCN-amplification status in the advanced stage patient data group. Previous research has shown that miR-9 upregulation correlates with breast cancer metastasis (Ma et al., 2010), supporting our finding of miR-9 as a new biomarker for advanced neuroblastoma. Furthermore, we also found miR-9 to be significantly upregulated in short-term survival patients within the advanced stage neuroblastoma group in other publicly available data (Scaruffi et al., 2009), confirming the validity of our finding. To increase the specificity of differentiating high-risk and short-term survival groups, further research is needed to identify additional miR-9 activating factors besides MYCN as well as the common biological pathways of miR-9 target genes in neuroblasts (Fig. 1). 


\section{MicroRNA and target gene signature in advanced neuroblastoma}

Expression levels of mRNA depend on various factors including DNA copy number, DNA methylation, histone acetylation, active transcription factors, splicing factors, and regulating miRNAs. Here we analyze mRNA expression in a miRNA-centric manner, comparing it with miRNA expression data to increase the specificity of advanced neuroblastoma signatures. This is based on our hypothesis that if a miRNA's function is important and causal to progress neuroblastoma and the expression of the miRNA is up (down)-regulated in the advanced stage, its targets will be preferentially down (up)regulated in the advanced neuroblastoma. Therefore, we will define a miRNA and its targets as important to the advanced neuroblastoma if such cases are found. Note that this method proceeds without bias of previous knowledge and simply searches for consistent expression data across different datasets (mRNA and miRNA expression), thus allowing novel findings.

The Vandesompele group, which has recently identified correlations between MYCNinduced miRNAs and poor outcome in MYCN-activated tumors (Mestdagh et al., 2010b), kindly provided us matching mRNA and miRNA expression patterns with an annotation file. We also downloaded the mRNA data, disease state, and MYCN amplification status from the Gene Express Omnibus (Series accession number GSE21713). The disease states followed the International Neuroblastoma Staging System's four stages. Stages 1 and 2 indicating the cancer is still localized, while stages 3 and 4 indicate metastasis of the original tumor. To understand global genetic differences in advanced neuroblastoma, we defined two groups for comparison: stage 4 as one group and stage 1 and 2 as another, regardless of MYCN amplification status. Stage 3 data were excluded in order to obtain maximal contrast between the non-metastatic stages and metastatic stage 4 . By comparing miRNA and mRNA expression of these two groups, we expected to obtain a more specific advanced neuroblastoma signature, which might not be apparent through mRNA or miRNA analysis alone.

\section{1 mRNA expression analysis}

\subsubsection{Differently expressed mRNAs in advanced neuroblastoma}

In total, there are 30 primary tumor sample data from 14 stage 4 patients and 16 stage 1 and 2 patients. Briefly, the Vandesomepele group derived the expression data as follows: after each sample expression dataset was obtained using GeneChip Human Exon 1.0 ST Arrays (Affymetrix), all exon data were combined to transcript clusters (hg18/core exons), to obtain expression information per gene after normalization according to the RMA-sketch algorithm using Affymetrix Power Tools. We used these RMA normalized data calculated by the Vandesomepele group to obtain differentially expressed genes between the two groups. Student t-tests were performed using Microsoft Excel functions and mRNA lists with pvalues less than 0.05 were prepared as up- and down-regulated mRNAs using HUGO Gene Nomenclature Committe (HGNC) gene symbol annotation. We ignored transcripts without gene symbol annotation or empty data points among patient samples. If more than two probe sets corresponded to one gene symbol, we chose the probe set with lower p-values. Among a total 372 up-regulated and 689 down-regulated mRNAs with gene symbols having $\mathrm{p}<0.05$, the top 50 genes with the greatest fold changes are shown in Table 1 . 
Neuroblastoma - Present and Future

\begin{tabular}{|c|c|c|c|c|c|}
\hline $\begin{array}{c}\text { Down- } \\
\text { Regulated Gene }\end{array}$ & p-value & $\begin{array}{c}\text { Log2 (Fold } \\
\text { Change) }\end{array}$ & $\begin{array}{l}\text { Up-Regulated } \\
\text { Gene }\end{array}$ & p-value & $\begin{array}{c}\text { Log2(Fold } \\
\text { Change) }\end{array}$ \\
\hline ALCAM & 0.012519 & -1.1404 & ACTA2 & 0.017706 & 0.84008 \\
\hline APBA1 & 0.000975 & -1.00703 & BIRC5 & 0.007473 & 0.88797 \\
\hline АТР2B4 & 0.008721 & -1.02324 & CCNA2 & 0.009386 & 0.79357 \\
\hline CADM3 & 0.002402 & -1.24665 & CDC45L & 0.007403 & 0.75931 \\
\hline CDH6 & 0.049003 & -1.01205 & CDCA5 & 0.008474 & 0.77314 \\
\hline DOC2B & 0.013732 & -1.02395 & CMBL & 0.004642 & 0.72441 \\
\hline DRD2 & 0.001234 & -1.08816 & DDX1 & 0.046072 & 1.22142 \\
\hline ECEL1 & 0.003074 & -1.10837 & E2F3 & 0.000912 & 0.74161 \\
\hline EPB41L3 & 0.008477 & -1.37384 & FN1 & 0.008058 & 1.03848 \\
\hline HIST1H1A & 0.016193 & -1.34667 & FOXM1 & 0.008946 & 0.9346 \\
\hline HS6ST3 & 0.000384 & -1.10939 & GJC1 & 0.002895 & 0.82971 \\
\hline LRRTM4 & 0.040988 & -1.31354 & HIST1H2BM & 0.037272 & 0.85818 \\
\hline NRCAM & 0.031584 & -1.04967 & HMGB2 & 0.006813 & 0.883 \\
\hline NTRK1 & 0.001072 & -1.79752 & MAD2L1 & 0.012955 & 0.92231 \\
\hline PGM2L1 & 0.010537 & -1.14954 & MYBL2 & 0.019576 & 0.82346 \\
\hline PLXNC1 & 0.000939 & -1.08949 & MYCN & 0.019589 & 1.23078 \\
\hline PMP22 & 0.003898 & -1.62031 & NAG & 0.020134 & 1.26792 \\
\hline PRKCA & 0.011104 & -1.04588 & ODC1 & 0.001326 & 1.3045 \\
\hline PRPH & 0.003777 & -1.41955 & PAICS & 0.000609 & 0.74121 \\
\hline PTN & 0.00069 & -1.19001 & PHGDH & 0.026183 & 0.88181 \\
\hline RAB3C & 0.025466 & -1.00737 & RRM2 & 0.00773 & 1.46016 \\
\hline REEP1 & 0.002232 & -1.00576 & SLC16A1 & 0.000721 & 0.93447 \\
\hline SCG2 & 0.009003 & -1.21159 & TYMS & 0.016238 & 0.95479 \\
\hline SCN9A & 0.0207 & -1.02774 & VCAN & 0.019634 & 0.99934 \\
\hline SYN3 & 0.006658 & -1.04893 & & & \\
\hline TMEM176A & 0.006741 & -1.11877 & & & \\
\hline
\end{tabular}

Table 1 . Top 50 genes with the greatest fold changes among differently expressed mRNAs in advanced neuroblastoma ( $\mathrm{p}<0.05)$. 


\subsubsection{Mapping differentiated mRNAs to regulating miRNAs}

For all mRNAs identified as differentially regulated, their regulating miRNAs were predicted using the miBridge miRNA target prediction method (v.1).We then calculated enrichment scores for miRNAs based on their target enrichment tests among the differentially regulated mRNAs. Score over 0 means that the predicted miRNA is significant in the system, 1 being optimal. Table 2 shows the predicted regulating miRNAs (within the miRNA list in the array measured) with target enrichment score over 0. Within the mir-1792 cluster, hsa-miR-18* targets are enriched in the down-regulated mRNAs, supporting our hypothesis and miRNA target predictions (though miR-92a is not included in this $p<0.05$ list, inclusion of genes with less than 15 empty values in patient samples yields a miR-92a score of 0.04 and the prediction that it is up-regulated in advanced neuroblastoma

\begin{tabular}{lcc}
\hline \multicolumn{1}{c}{ Regulating miRNA } & $\begin{array}{c}\text { Enrichment } \\
\text { score }\end{array}$ & Predicted as \\
\hline hsa-miR-9 & 0.03642 & up \\
\hline hsa-miR-18a* & 0.3787 & up \\
\hline hsa-miR-136 & 0.3765 & up \\
\hline hsa-miR-152 & 0.03642 & up \\
\hline hsa-miR-185 & 0.7552 & up \\
\hline hsa-miR-205 & 0.49874 & up \\
\hline hsa-miR-214 & 0.46397 & up \\
\hline hsa-miR-221 & 0.73754 & up \\
\hline hsa-miR-324-3p & 0.49392 & up \\
\hline hsa-miR-326 & 0.97012 & up \\
\hline hsa-miR-328 & 0.86602 & up \\
\hline hsa-miR-346 & 0.51208 & up \\
\hline hsa-miR-500 & 0.03642 & up \\
\hline hsa-miR-610 & 0.08688 & up \\
\hline hsa-miR-650 & 0.81604 & up \\
\hline hsa-miR-489 & & \\
\hline
\end{tabular}

Table 2. Predicted miRNAs as potential regulators of advanced neuroblastoma (enrichment score $>0$ ).

\section{2 miRNA expression analysis}

The miRNA expression data were obtained from the same patient samples analyzed in the mRNA expression: total 14 stage 4 patients and 16 stage 1 and 2 patients. We used the RMA normalized data with 312 mature miRNA annotations as provided by the Vandesomepele group. Student t-test was performed using Microsoft Excel functions; miRNA lists of score $>0$ are shown in Table 3. 


\begin{tabular}{|c|c|c|c|c|c|}
\hline $\begin{array}{l}\text { up-regulated } \\
\text { miRNA }\end{array}$ & p-value & $\begin{array}{l}\log _{2} \text { (fold } \\
\text { change) }\end{array}$ & $\begin{array}{c}\text { down-regulated } \\
\text { miRNA }\end{array}$ & p-value & $\begin{array}{c}\log _{2} \text { (fold } \\
\text { change) }\end{array}$ \\
\hline hsa-miR-9 & 0.037541 & 1.173707 & hsa-miR-15a & 0.044916 & -0.49254 \\
\hline hsa-miR-9* & 0.018107 & 1.235894 & hsa-miR-24 & 0.030286 & -0.44341 \\
\hline hsa-miR-18a & 0.02853 & 0.570961 & hsa-miR-26a & 0.007846 & -0.53493 \\
\hline hsa-miR-18a* & 0.001933 & 0.58714 & hsa-miR-26b & 0.011224 & -0.44942 \\
\hline hsa-miR-19a & 0.038493 & 0.588543 & hsa-miR-30a-3p & 0.023514 & -0.68173 \\
\hline hsa-mir-92 & 0.006916 & 0.782683 & hsa-miR-30b & 0.00698 & -0.50628 \\
\hline hsa-miR-105 & 0.036113 & 1.681312 & hsa-miR-30e-3p & 0.023054 & -0.77332 \\
\hline hsa-miR-320 & 0.023005 & 0.362074 & hsa-miR-95 & 0.012503 & -0.91388 \\
\hline hsa-miR-375 & 0.03906 & 1.901862 & hsa-miR-103 & 0.0395 & -0.35021 \\
\hline hsa-miR-517a & 0.045293 & 1.423221 & hsa-miR-125b & 0.00271 & -0.89665 \\
\hline hsa-miR-520g & 0.024751 & 1.757529 & hsa-miR-128a & 0.016545 & -0.73381 \\
\hline hsa-miR-526b* & 0.049281 & 0.601139 & hsa-miR-137 & 0.000167 & -1.78508 \\
\hline \multirow[t]{17}{*}{ hsa-miR-645 } & 0.014262 & 0.758181 & hsa-miR-140 & 0.010729 & -0.3409 \\
\hline & & & hsa-miR-148b & 0.009921 & -0.56818 \\
\hline & & & hsa-miR-149 & 0.002849 & -1.13549 \\
\hline & & & hsa-miR-190 & 0.048211 & -1.23233 \\
\hline & & & hsa-miR-204 & 0.008341 & -2.21371 \\
\hline & & & hsa-miR-215 & 0.005819 & -1.68041 \\
\hline & & & hsa-miR-216 & 0.042686 & -1.38497 \\
\hline & & & hsa-miR-218 & 0.045402 & -0.86703 \\
\hline & & & hsa-miR-324-3p & 0.034135 & -0.52073 \\
\hline & & & hsa-miR-324-5p & 0.028667 & -0.74833 \\
\hline & & & hsa-miR-326 & 0.048664 & -0.71389 \\
\hline & & & hsa-miR-330 & 0.010354 & -0.98399 \\
\hline & & & hsa-miR-331 & 0.004691 & -0.73239 \\
\hline & & & hsa-miR-340 & 0.010086 & -0.77428 \\
\hline & & & hsa-miR-488 & 0.01028 & -1.08969 \\
\hline & & & hsa-miR-491 & 0.042597 & -0.68084 \\
\hline & & & hsa-miR-628 & 0.000553 & -1.00498 \\
\hline
\end{tabular}

Table 3. Differentially expressed miRNAs in the advanced neuroblastoma (student t-test $\mathrm{p}<$ $0.05)$

We found that two miRNAs predicted as up-regulated with enrichment score $>0$, miR-9 and miR-18a*, are actually up-regulated in microarray experiments with student t-test $\mathrm{p}<$ 0.05. Since miR-18a* is a minor strand (less present than miR-18a) within the mir-17-92 cluster, we conclude that miR-9 is the most consistent miRNA in terms of its expression and its targets' expression in our analysis. Fig. 2 shows the box plots of miR-9 expressions in advanced and low-risk stage patient samples together with five other miRNAs whose expression values are most significantly changed in up- and down-regulated miRNAs. 

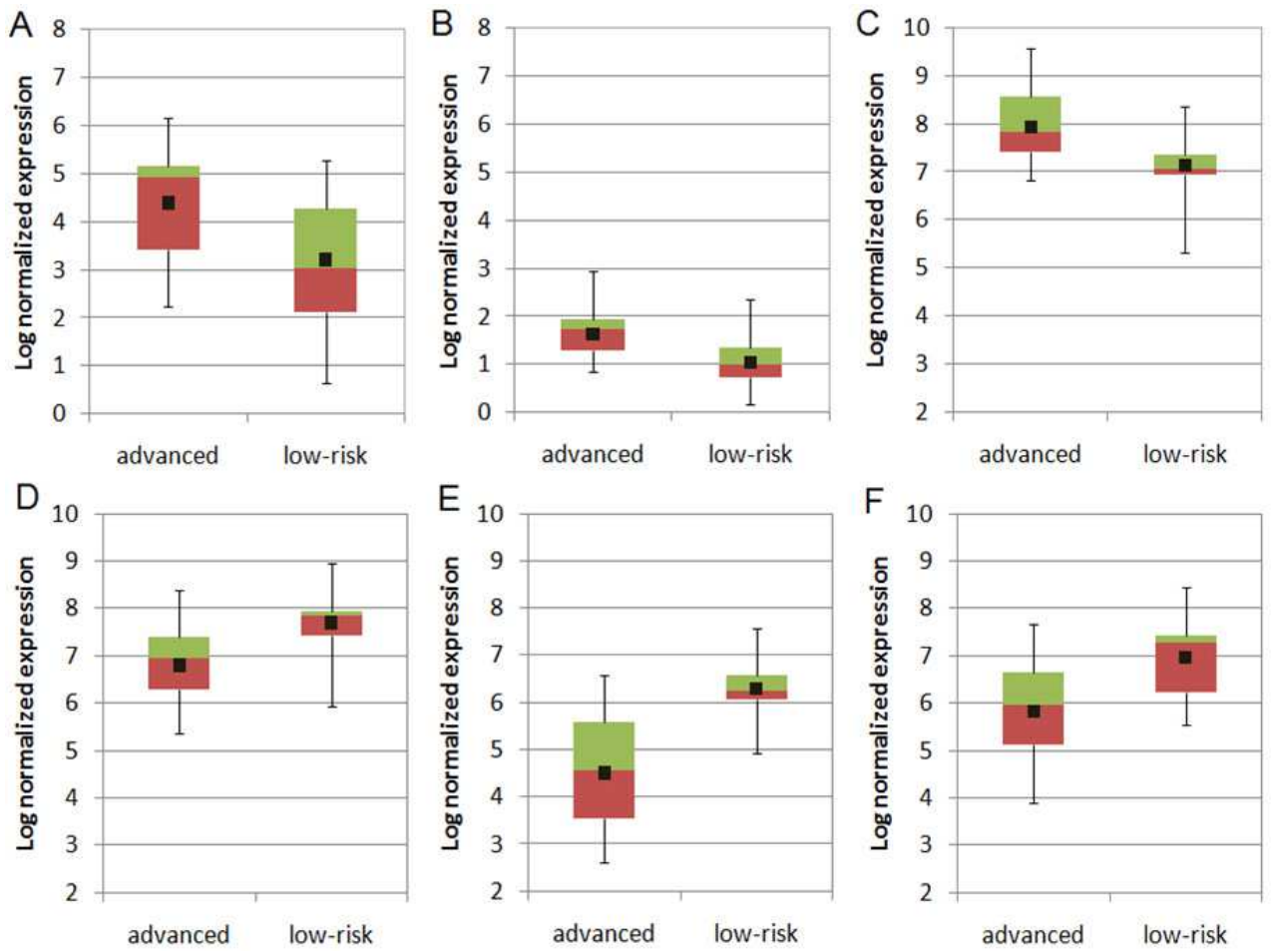

Fig. 2. Box plots of miR-9 (A), miR-18a* (B), miR-92 (C), miR-125b (D), miR-137 (E), and miR149 (F) expression between advanced and low-risk stage neuroblastoma patient samples.

\subsection{Correlation of mRNA and miRNA expression data}

We found miR-9 to have the most consistent signal in terms of miRNA and mRNA expression level changes in advanced neuroblastoma. Note that miRNA expression pattern alone does not justify further in-depth analyses of miR-9 since other miRNAs have higher fold changes or lower p-value. Our approach thus provides a new way to prioritize important miRNAs. Interestingly, miR-9's seven predicted targets genes are found only in the down-regulated genes. However, this does not mean that expressions of miR-9 and its target genes are negatively correlated across entire samples. Fig. 3 shows the Pearson product moment correlation coefficient of miR-9 and its seven target genes across the 30 patient samples. For context, correlations between miR-9 and all mRNA expression are also shown. The correlations of miR-9 and target genes stand out from all other correlations, six of the seven targets being negatively correlated with miR-9. The six negatively correlated predicted targets are AGPAT4, BTBD9, GABBR1, KCNK10, LRRTM4, and S100PBP. Among them, LRRTM4 is in Table 1, containing top 50 greatest fold change genes. Fig. 4 shows the scatter plots of the two most negatively correlated miR-9 target genes.

Though miR-9 can target multiple genes at the same time, the expression of each target gene varies from person to person, so that its function on each target gene might vary 
among patients. In our analysis, six genes are predicted as functional targets in the neuroblastoma, potentially responsible for disease progression. As an example, some outliers shown in the circle in Fig. 4B are no longer outliers in the S100PBP case in Fig. 4A. Therefore, rather than one miRNA or one miRNA and its target, collective miR-9 targets and miR-9 might allow more accurate prediction of whether a neuroblastoma will progress to advanced stage.

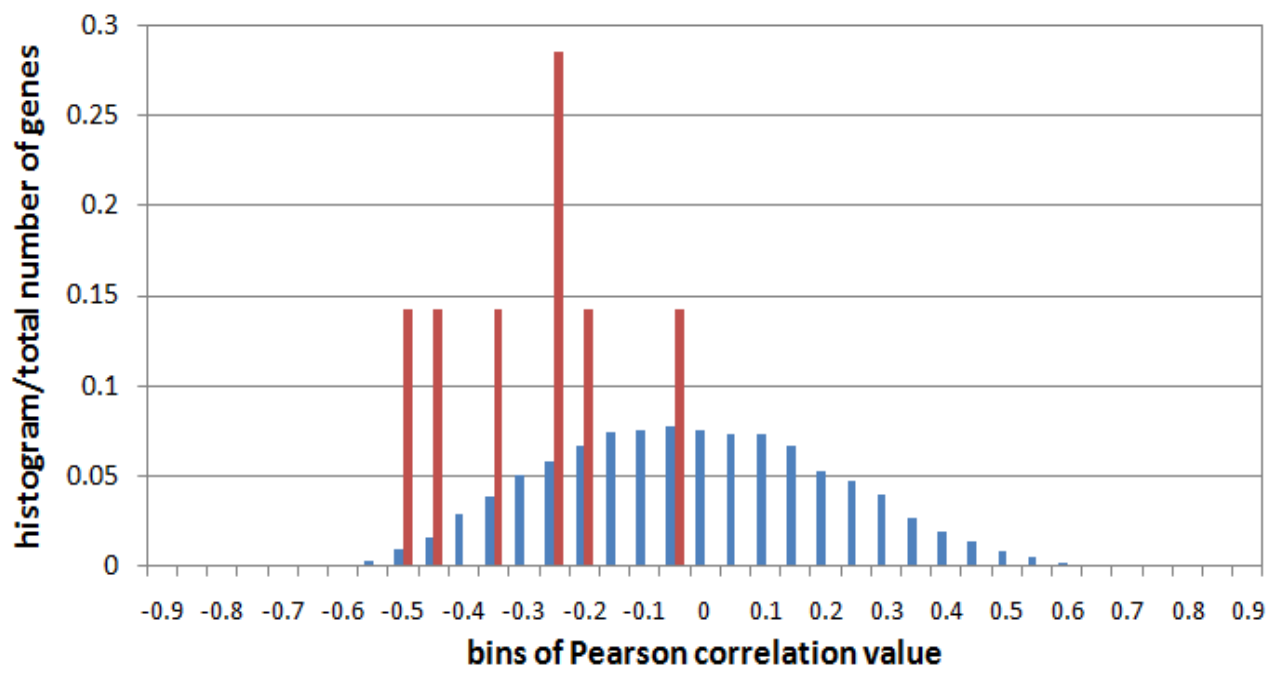

Fig. 3. Histogram of Pearson correlation values between miR-9 and gene expressions. The red bars show miR-9 target genes found in the down-regulated genes; blue bars represent all genes measured in the microarray experiments (total 1,4159 genes excluding ones with blank data $>5$ among samples).
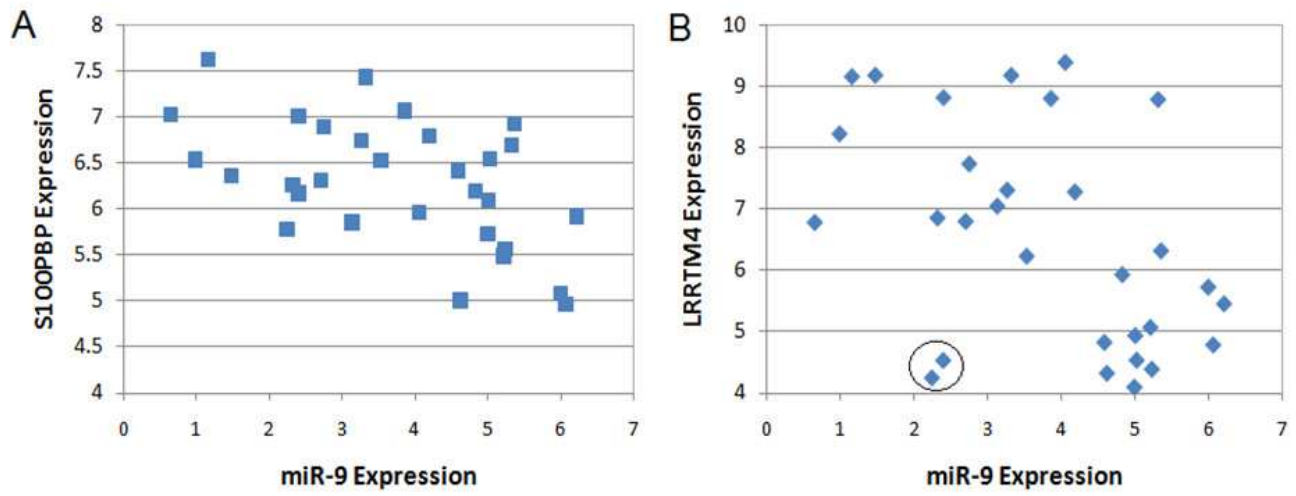

Fig. 4. Scatter plots showing miR-9 and target gene expressions for 30 patient samples. Pearson correlation coefficient of miR-9 and S100PBP is -0.53 (A) and that of miR-9 and LRRTM4 is -0.48 (B). 


\section{3. miR-9 signature in short - and long - lived patients}

Scaruffi et al. published research on non-coding RNA expressions with regard to outcome in high-risk neuroblastoma (Scaruffi et al., 2009). Among 31 high-risk, stage 4 neuroblastoma samples, miRNA expressions came from 17 short-term survivors (dead within 36 months from diagnosis) and 14 long-term survivors (alive with an overall survival time > 36 months) were deposited in GEO (Series accession number GSE16444). We downloaded the data, which were $\log _{2}$-transformed and quantile normalized, after obtaining the raw data using miRNA microarray System protocol v. 1.5 (Agilent Tenchnologies). Since they removed probes with less than $50 \%$ of detected slops across all arrays, many low signals were not present. We used the downloaded data to identify differential miRNAs between short- and long-term survivor groups. Significance was calculated with a student's t-test (unpaired, two-tailed, unequal variance); miRNAs upand down-regulated in short-term survivors with p-values less than 0.05 are shown in Table 4.

\begin{tabular}{lll|lll}
\hline $\begin{array}{c}\text { up-regulated } \\
\text { miRNA }\end{array}$ & p-value & $\begin{array}{c}\text { Log }_{2} \text { (fold } \\
\text { change) }\end{array}$ & \multicolumn{1}{|c}{$\begin{array}{c}\text { down- } \\
\text { regulated } \\
\text { miRNA }\end{array}$} & p-value & $\begin{array}{c}\text { Log }_{2} \text { (fold } \\
\text { change) }\end{array}$ \\
\hline hsa-miR-9 & 0.0377472 & 0.68625181 & hsa-miR-22 & 0.040065 & -0.990610191 \\
\hline hsa-miR-210 & 0.0284823 & 1.147684424 & hsa-miR-139-3p & 0.0478034 & -0.803202819 \\
\hline hsa-miR-425 & 0.0127439 & 0.62221312 & hsa-miR-181c* & 0.0497988 & -1.015817709 \\
\hline hsa-miR-758 & 0.0080157 & 1.01630988 & hsa-miR-302a & 0.0338064 & -0.915468934 \\
\hline hsa-miR-885-5p & 0.0117879 & 1.125425787 & hsa-miR-502-3p & 0.0481067 & -0.69939469 \\
\hline hsa-miR-885-3p & 0.0118758 & 0.669348934 & hsa-miR-886-3p & 0.0424204 & -1.691328238 \\
\hline hsa-miR-877 & 0.0276158 & 0.571214174 & & & \\
\hline hsa-miR-936 & 0.0388439 & 0.656436824 & & & \\
\hline
\end{tabular}

Table 4. Differentially expressed miRNAs in short-term survivors compared with long-term survivors. All patients had advanced neuroblastoma.

Due to the distinct difference between diseased and healthy status, the overall number of differentially expressed miRNAs with a certain p-value cutoff may be much greater than that within the disease group. Within the disease group, though neuroblastoma has diverse outcomes, differentiating stages within the neuroblastoma is more difficult than differentiating disease from normal. To increase differentiating power, we did not include stage 3 neuroblastoma data in our section 2 analysis. Here, we further differentiate a narrower range within stage 4 . Therefore, it is not surprising that fewer miRNAs were differentiated here than between advanced and low-risk neuroblastoma. Also, it is common for different research groups to produce differing lists of significantly changed genes. 
However, if underlying causes exist for advanced neuroblastoma which further lead to short-term survival, we might identify them from a persistent signal in various contexts. We find miR-9, the only miRNA common between Table 3 and 4 , to be such a consistent signal. We want to emphasize that miR-9 must be evaluated in connection with target gene expressions. It was found to be the most consistent miRNA in terms of down-regulating target mRNAs when induced. Moreover, its expression differs between short- and long-term survivors. When it comes to identifying miRNA signature from expression data, it is therefore crucial to compare a miRNA's target expression patterns with its own expression. While many studies focus on global correlations between miRNA and mRNA expressions without a clear regulating matrix, our method of assessing consistency clearly helps pinpoint the signature miRNA in the disease progression.
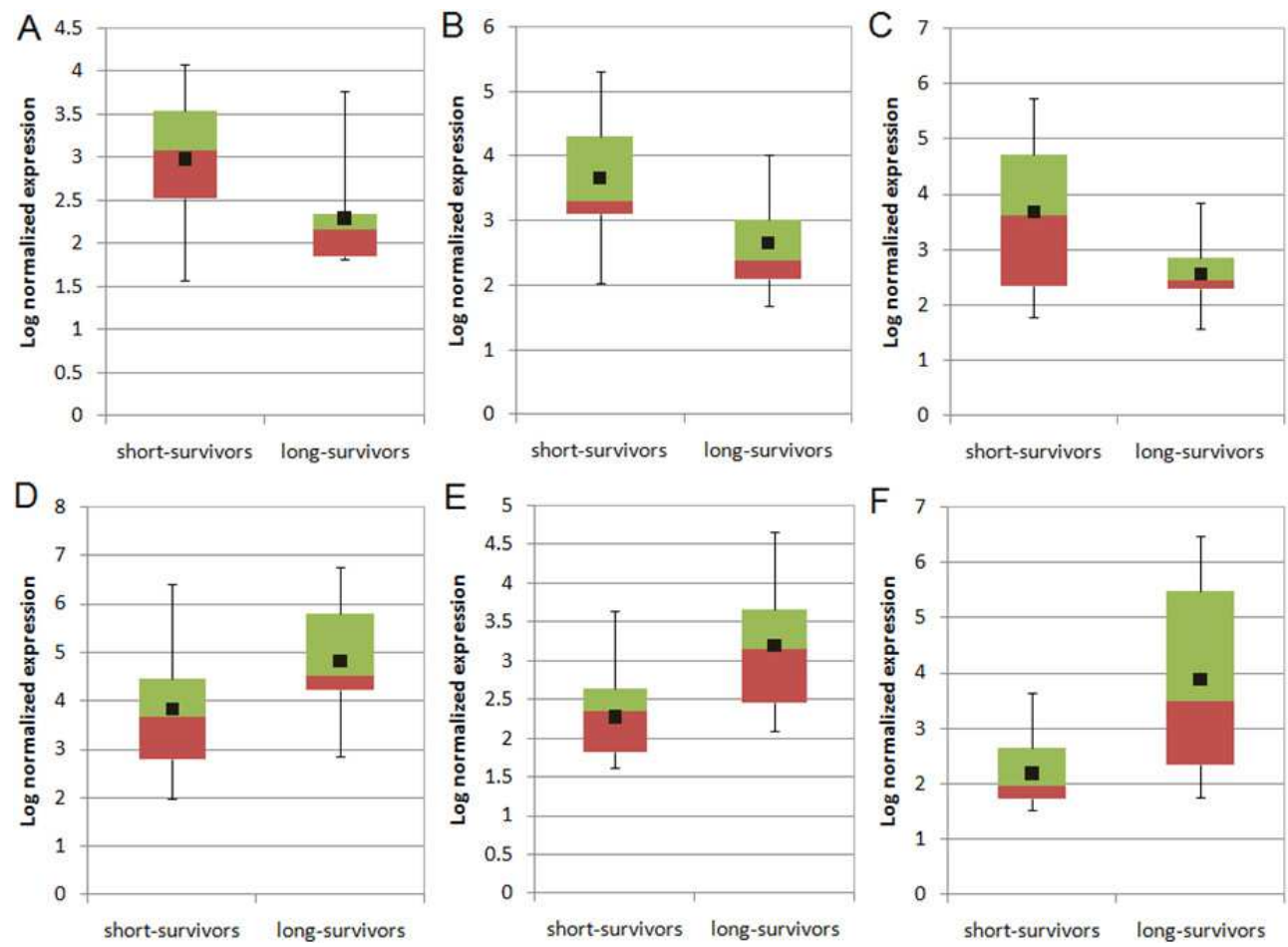

Fig. 5. Box plots of miR-9 (A), miR-758 (B), miR-885-5p (C), miR-22 (D), miR-302a (E), and miR-886-3p (F) expressions of neuroblastoma between short- and long-term survivors in advanced stage patients.

Fig. 5 shows the box plot of miR-9 and five other miRNA expressions (chosen in order of smaller p-values) of samples from short- and long-term survivors in the Table 4 . Though the p-value of miR-9 is not as low as others, its differentiating power is close to that of miR-758. In Fig. 2, the miR-9 level itself may not be powerful enough to differentiate advanced stage from low-risk neuroblastoma. Here, the data suggest that the broader range of miR-9 expression in advanced stage is due to its differentiating power between two sub-groups of 
advanced stage patients (absolute number of normalized data in each dataset is not meaningful). In terms of early prognostic power, miR-9 and its targets hold high promise. Further investigation using larger samples is needed.

\section{4. miR-9 functional model in advanced neuroblastoma}

Recently, Ma et al. reported that miR-9 directly targets E-cadherin (CDH1), leading to increased cell motility and invasiveness in breast cancer (Ma et al., 2010). Though in a different cell type, since the function of $\mathrm{CDH} 1$ in solid tumors is the same, we expect miR-9 to function similarly in the neuroblastoma. Moreover, as a MYCN-activated miRNA, miR-9 fits well within the neuroblastoma miRNA networks (Fig. 1) as a function of developing advanced metastasized neuroblastoma. Our identification of miR-9 and its targets thus makes sense functionally as an early prognostic marker for developing high mortality neuroblastoma. Fig. 1 includes miR-9 and CDH1 as a functional model of developing advanced stage neuroblastoma.

\section{Conclusion}

We have used our new method to analyze mRNA and miRNA expression data to identify signature miRNA and target genes in stage 4 neuroblastoma. To obtain maximal contrast between the non-metastatic stages and metastatic stage 4, we excluded stage 3 data and combined stage 1 and 2 as low-risk. When we compared advanced and low-risk stage neuroblastoma, miR-9 related expressions had the most consistent data between mRNA and miRNA expression. We also confirmed that six out of the seven predicted targets were negatively correlated with miR-9 expression across entire samples. Furthermore, miR-9 expression was significantly up-regulated in samples from short-term survivors compared with those from long-term survivors within the same advanced-stage neuroblastoma group. In addition to these data-driven analyses, note that miR-9 has been identified as inducing metastasis in breast cancer by targeting E-cadherin. Therefore, our claim of expressions of miR-9 and its targets as a signature of advancing neuroblastoma fits well with previous studies. Further investigation with a larger number of samples is needed.

\section{Acknowledgment}

We thank the Vandesompele group for providing paired mRNA and miRNA expression data of samples from neuroblastoma patients.

\section{References}

Ajay, S. S., Athey, B. D., \& Lee, I. (2010). Unified translation repression mechanism for microRNAs and upstream AUGs. BMC Genomics, Vol. 11, pp. (155)

Asgharzadeh, S., Pique-Regi, R., Sposto, R., Wang, H., et al. (2006). Prognostic significance of gene expression profiles of metastatic neuroblastomas lacking MYCN gene amplification. J Natl Cancer Inst, Vol. 98, No. 17, pp. (1193-203) 
Bordow, S. B., Norris, M. D., Haber, P. S., Marshall, G. M., et al. (1998). Prognostic significance of MYCN oncogene expression in childhood neuroblastoma. J Clin Oncol, Vol. 16, No. 10, pp. (3286-94)

Bray, I., Tivnan, A., Bryan, K., Foley, N. H., et al. (2011). MicroRNA-542-5p as a novel tumor suppressor in neuroblastoma. Cancer Lett, Vol. 303, No. 1, pp. (56-64)

Buechner, J., Tomte, E., Haug, B. H., Henriksen, J. R., et al. (2011). Tumour-suppressor microRNAs let-7 and mir-101 target the proto-oncogene MYCN and inhibit cell proliferation in MYCN-amplified neuroblastoma. Br J Cancer, Vol. 105, No. 2, pp. (296-303)

Chen, Y., \& Stallings, R. L. (2007). Differential patterns of microRNA expression in neuroblastoma are correlated with prognosis, differentiation, and apoptosis. Cancer Res, Vol. 67, No. 3, pp. (976-83)

Christoffersen, N. R., Shalgi, R., Frankel, L. B., Leucci, E., et al. (2010). p53-independent upregulation of miR-34a during oncogene-induced senescence represses MYC. Cell Death Differ, Vol. 17, No. 2, pp. (236-45)

De Preter, K., Vermeulen, J., Brors, B., Delattre, O., et al. (2010). Accurate outcome prediction in neuroblastoma across independent data sets using a multigene signature. Clin Cancer Res, Vol. 16, No. 5, pp. (1532-41)

Foley, N. H., Bray, I., Watters, K. M., Das, S., et al. (2011). MicroRNAs 10a and 10b are potent inducers of neuroblastoma cell differentiation through targeting of nuclear receptor corepressor 2. Cell Death Differ, Vol. 18, No. 7, pp. (1089-98)

Foley, N. H., Bray, I. M., Tivnan, A., Bryan, K., et al. (2010). MicroRNA-184 inhibits neuroblastoma cell survival through targeting the serine/threonine kinase AKT2. Mol Cancer, Vol. 9, pp. (83)

Guo, J., Dong, Q., Fang, Z., Chen, X., et al. (2010). Identification of miRNAs that are associated with tumor metastasis in neuroblastoma. Cancer Biol Ther, Vol. 9, No. 6, pp. (446-52)

Haug, B. H., Henriksen, J. R., Buechner, J., Geerts, D., et al. (2011). MYCN-regulated miRNA-92 inhibits secretion of the tumor suppressor DICKKOPF-3 (DKK3) in neuroblastoma. Carcinogenesis, Vol. 32, No. 7, pp. (1005-12)

He, L., He, X., Lim, L. P., de Stanchina, E., et al. (2007). A microRNA component of the p53 tumour suppressor network. Nature, Vol. 447, No. 7148, pp. (1130-4)

He, L., Thomson, J. M., Hemann, M. T., Hernando-Monge, E., et al. (2005). A microRNA polycistron as a potential human oncogene. Nature, Vol. 435, No. 7043, pp. (82833)

Heuberger, J., \& Birchmeier, W. (2010). Interplay of cadherin-mediated cell adhesion and canonical Wnt signaling. Cold Spring Harb Perspect Biol, Vol. 2, No. 2, pp. (a002915)

Jiang, M., Stanke, J., \& Lahti, J. M. (2011). The connections between neural crest development and neuroblastoma. Curr Top Dev Biol, Vol. 94, pp. (77-127)

Kim, N. H., Kim, H. S., Li, X.-Y., Lee, I., et al. (2011). A p53/miRNA-34 Axis Regulates Snail1-Dependent Cancer Cell Epithelial-Mesenchymal Transition. J Cell Biol, Vol. 195, No. 3, pp. (417-433) 
Kim, V. N. (2005). MicroRNA biogenesis: coordinated cropping and dicing. Nat Rev Mol Cell Biol, Vol. 6, No. 5, pp. (376-85)

Kozomara, A., \& Griffiths-Jones, S. (2010). miRBase: integrating microRNA annotation and deep-sequencing data. Nucleic Acids Res, Vol. 39, No. Database issue, pp. (D152-7)

Lee, I., Ajay, S. S., Yook, J. I., Kim, H. S., et al. (2009). New class of microRNA targets containing simultaneous 5'-UTR and 3'-UTR interaction sites. Genome Res, Vol. 19, No. 7, pp. (1175-83)

Lim, L. P., Lau, N. C., Garrett-Engele, P., Grimson, A., et al. (2005). Microarray analysis shows that some microRNAs downregulate large numbers of target mRNAs. Nature, Vol. 433, No. 7027, pp. (769-73)

Lin, R. J., Lin, Y. C., Chen, J., Kuo, H. H., et al. (2010). microRNA signature and expression of Dicer and Drosha can predict prognosis and delineate risk groups in neuroblastoma. Cancer Res, Vol. 70, No. 20, pp. (7841-50)

Ma, L., Young, J., Prabhala, H., Pan, E., et al. (2010). miR-9, a MYC/MYCN-activated microRNA, regulates E-cadherin and cancer metastasis. Nat Cell Biol, Vol. 12, No. 3, pp. (247-56)

Matsubara, H., Takeuchi, T., Nishikawa, E., Yanagisawa, K., et al. (2007). Apoptosis induction by antisense oligonucleotides against miR-17-5p and miR-20a in lung cancers overexpressing miR-17-92. Oncogene, Vol. 26, No. 41, pp. (6099-105)

Mestdagh, P., Bostrom, A. K., Impens, F., Fredlund, E., et al. (2010a). The miR-17-92 microRNA cluster regulates multiple components of the TGF-beta pathway in neuroblastoma. Mol Cell, Vol. 40, No. 5, pp. (762-73)

Mestdagh, P., Fredlund, E., Pattyn, F., Schulte, J. H., et al. (2010b). MYCN/c-MYC-induced microRNAs repress coding gene networks associated with poor outcome in MYCN/c-MYC-activated tumors. Oncogene, Vol. 29, No. 9, pp. (1394-404)

Oberthuer, A., Berthold, F., Warnat, P., Hero, B., et al. (2006). Customized oligonucleotide microarray gene expression-based classification of neuroblastoma patients outperforms current clinical risk stratification. J Clin Oncol, Vol. 24, No. 31, pp. (5070-8)

Oberthuer, A., Hero, B., Berthold, F., Juraeva, D., et al. (2010). Prognostic impact of gene expression-based classification for neuroblastoma. J Clin Oncol, Vol. 28, No. 21, pp. (3506-15)

Papadopoulos, G. L., Reczko, M., Simossis, V. A., Sethupathy, P., et al. (2009). The database of experimentally supported targets: a functional update of TarBase. Nucleic Acids Res, Vol. 37, No. Database issue, pp. (D155-8)

Scaruffi, P., Stigliani, S., Moretti, S., Coco, S., et al. (2009). Transcribed-Ultra Conserved Region expression is associated with outcome in high-risk neuroblastoma. BMC Cancer, Vol. 9, pp. (441)

Schulte, J. H., Horn, S., Otto, T., Samans, B., et al. (2008). MYCN regulates oncogenic MicroRNAs in neuroblastoma. Int J Cancer, Vol. 122, No. 3, pp. (699-704)

Tivnan, A., Foley, N. H., Tracey, L., Davidoff, A. M., et al. (2010). MicroRNA-184-mediated inhibition of tumour growth in an orthotopic murine model of neuroblastoma. Anticancer Res, Vol. 30, No. 11, pp. (4391-5) 
Tivnan, A., Tracey, L., Buckley, P. G., Alcock, L. C., et al. (2011). MicroRNA-34a is a potent tumor suppressor molecule in vivo in neuroblastoma. BMC Cancer, Vol. 11, pp. (33) 


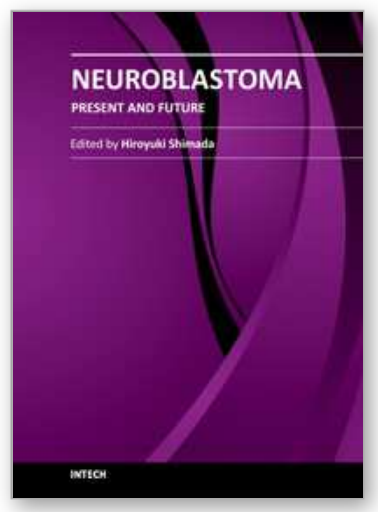

\author{
Neuroblastoma - Present and Future \\ Edited by Prof. Hiroyuki Shimada
}

ISBN 978-953-307-016-2

Hard cover, 366 pages

Publisher InTech

Published online 08, February, 2012

Published in print edition February, 2012

Neuroblastoma, once called "enigmatic", due to "unpredictable" clinical behaviors, is composed of biologically diverse tumors. Molecular/genomic properties unique to the individual tumors closely link to the clinical outcomes of patients. Establishing risk stratification models after analyzing biologic characteristics of each case has made a great success in patient management. However, the trend of improving survival rates in neuroblastoma over the last 30 years has started to level off, and currently available treatment modalities have almost reached to their maximized intensity. Furthermore, aggressive treatment causes significant long-term morbidities to the survivors. We really need to make the next step to the level of personalized medicine with more precise understanding of neuroblastoma biology. This book includes useful data and insights from the world's experts in this field. I believe this book can make an excellent contribution to all the investigators working hard and fighting for the children stricken by this disease.

\title{
How to reference
}

In order to correctly reference this scholarly work, feel free to copy and paste the following:

Victoria R. Chu and Inhan Lee (2012). MicroRNA Target Signatures in Advanced Stage Neuroblastoma, Neuroblastoma - Present and Future, Prof. Hiroyuki Shimada (Ed.), ISBN: 978-953-307-016-2, InTech, Available from: http://www.intechopen.com/books/neuroblastoma-present-and-future/microrna-targetsignatures-in-advanced-stage-neuroblastoma

\section{INTECH}

open science | open minds

\section{InTech Europe}

University Campus STeP Ri

Slavka Krautzeka 83/A

51000 Rijeka, Croatia

Phone: +385 (51) 770447

Fax: +385 (51) 686166

www.intechopen.com

\section{InTech China}

Unit 405, Office Block, Hotel Equatorial Shanghai

No.65, Yan An Road (West), Shanghai, 200040, China

中国上海市延安西路65号上海国际贵都大饭店办公楼 405 单元

Phone: +86-21-62489820

Fax: $+86-21-62489821$ 
(C) 2012 The Author(s). Licensee IntechOpen. This is an open access article distributed under the terms of the Creative Commons Attribution 3.0 License, which permits unrestricted use, distribution, and reproduction in any medium, provided the original work is properly cited. 